\title{
GROUND TOPOGRAPHY ESTIMATION OVER FORESTS CONSIDERING POLARIMETRIC SAR INTERFEROMETRY
}

\author{
C. López-Martínez, A. Alonso, X. Fàbregas* \\ Universitat Politècnica de Catalunya \\ Signal Theory and Communications Dep. \\ Jordi Girona,1-3, 08034, Barcelona, Spain \\ Email: Carlos.Lopez@tsc.upc.edu
}

\begin{abstract}
The work detailed in this paper analyzes the topographic phase retrieval process on forested areas by means of Polarimetric Interferometric SAR data. On the basis of the Random Volume over Ground scattering model, an alternative implementation for the retrieval of the topographic phase, avoiding the bias introduced by the volumetric scattering components is presented.
\end{abstract}

Index Terms - Polarimetric SAR Interferometry, Ground topography estimation.

\section{INTRODUCTION}

Forest areas cover approximately $30 \%$ of the Earth's solid surface, with a mean tree height of about $20 \mathrm{~m}$. Any attempt to provide global surface mapping based on SAR Interferometry (InSAR) is affected by the presence of the vegetation cover, in such a way, that the interferometric phase due to the ground surface scattering presents a bias, respect to the actual value, due to vegetation. The magnitude of this bias error depends on the system parameters, mainly the microwave frequency, and on the forest characteristics, basically the extinction coefficient. From a quantitative point of view, this error and may range up to the mean tree height.

The evaluation of volume decorrelation effects in multibaseline InSAR data has demonstrated that there is no conventional frequency, from $\mathrm{P}$ - up to X-band, able to be sensitive only to the ground under a vegetation layer without being affected by any volume, i.e., the vegetation scattering contribution. In consequence, all Digital Elevation Models (DEM's) generated by means of conventional InSAR are affected by a more or less significant vegetation bias. The correction of this inherent vegetation bias, and the estimation of the underlying ground topography is an essential improvement of the topographic information provided by InSAR, with great ecological as well as commercial impact.

*This work has been funded by the Ramon-y-Cajal program and the TEC project number TEC2007-65690 of the Science and Innovation Department of the Spanish government.

\author{
K. P. Papathannassiou
}

\author{
German Aerospace Center \\ Microwaves and Radar Institute \\ P.O. Box 1116, D-82230 Wessling, Germany \\ Email: Kostas.Papathanassiou@dlr.de
}

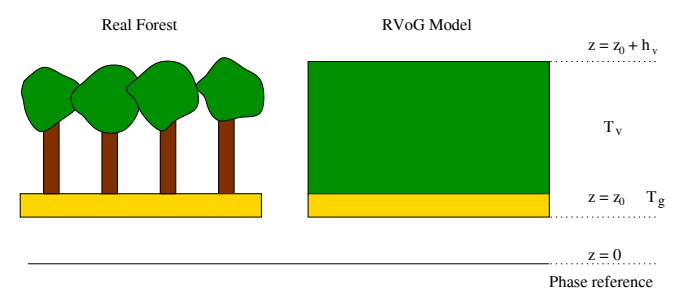

Fig. 1: Modeling of forest scattering by the RVoG model.

In this work, an alternative implementation of the Random Volume over Ground (RVoG) scattering model inversion to estimate the underlying ground topography from Polarimetric Interferometric SAR data (PolInSAR) [1] shall be presented. This new technique presents several advantages respect to the conventional use of the RVoG model, namely, the proposed approach presents a more robust, by means of parameter estimation, implementation and an unambiguous estimation of the ground topography.

\section{POLARIMETRIC SAR INTERFEROMETRY}

A PolInSAR acquisition scheme works on the basis of acquiring two fully polarimetric data sets from slightly different postions in space. In case of distributed scatterers, such forested areas, PolInSAR data are fully characterized by the coherency matrix

$$
\mathbf{T}_{6}=E\left\{\mathbf{k k}^{H}\right\}=\left[\begin{array}{ll}
\mathbf{T}_{11} & \boldsymbol{\Omega}_{12} \\
\boldsymbol{\Omega}_{12}^{H} & \mathbf{T}_{22}
\end{array}\right]
$$

where ${ }^{H}$ indicates complex transposition. The matrices $\mathbf{T}_{11}$ and $\mathbf{T}_{22}$ correspond to the individual polarimetric coherency matrices of the two passes and $\boldsymbol{\Omega}_{12}$ is the polarimetric interferometric coherency matrix.

In order to make possible the retrieval of quantitative information in case of forests, (1) is modeled according to a two-layer model, also known as RVoG model, see Fig. 1 [2]. The first layer, with a height $h_{v} \mathrm{~m}$ respect to a given reference $z_{0} \mathrm{~m}$ and a mean extinction coefficient $\sigma \mathrm{dB} / \mathrm{m}$, represents the 
volume scattering contribution of the forest canopy thought a set of randomly oriented particles. The polarimetric contribution of the volume scattering is represented by

$$
\mathbf{T}_{v}=m_{v}\left[\begin{array}{lll}
1 & 0 & 0 \\
0 & \eta & 0 \\
0 & 0 & \eta
\end{array}\right] 0 \leq \eta \leq 0.5
$$

where $m_{v}$ represents the volume scattering amplitude per unit volume and $\eta$ accounts for the mean particle shape, ranging from $\eta=0$ in case of spheres to $\eta=0.5$ in case of needle like or dipole particles. The second layer of the RVoG scattering model accounts for the ground scattering contribution, where the polarimetric contribution is modeled, under the hypothesis of reflection symmetry, according to the coherency matrix

$$
\mathbf{T}_{g}=m_{g}\left[\begin{array}{ccc}
1 & t_{12} & 0 \\
t_{12}^{*} & t_{22} & 0 \\
0 & 0 & t_{33}
\end{array}\right]
$$

where $m_{g}$ represents the ground scattering amplitude. It is worth to notice that the location of ground contribution is well located in the vertical dimension at a height $z_{0} \mathrm{~m}$, that in phase is represented by the term $\phi_{1}$ rad. On the contrary, the contribution of the volume scattering is more diffuse as it ranges from the bottom to the top of the canopy. The bottom limit of this canopy is represented, in terms of phase, by $\phi_{2} \mathrm{rad}$, that is is normally assumed to be equal to $\phi_{1} \mathrm{rad}$, whereas the top of the canopy is at a height $z_{0}+h_{v} \mathrm{~m}$, that will present the corresponding phase value. The height information, as measured by the PolInSAR sensor, is encoded in phase through the vertical wavenumber

$$
\kappa_{z}=\frac{\kappa \Delta \theta}{\sin \theta_{0}}=2 \frac{2 \pi}{\lambda} \frac{\Delta \theta}{\sin \theta_{0}}
$$

where the SAR system shall be supposed to operate at a wavelength $\lambda$ and in an interferometric configuration producing a baseline of $B \mathrm{~m}$, an incidence angle difference of $\Delta \theta \mathrm{rad}$ and a mean incidence angle $\theta_{0}$.

Under the assumption of the RVoG model to describe the forest scattering, the polarimetric matrices $\mathbf{T}_{11}$ and $\mathbf{T}_{22}$ are considered equal and modeled as follows

$$
\begin{aligned}
\mathbf{T}_{11} & =\mathbf{I}_{1}^{v}+e^{\frac{-2 \sigma h_{v}}{\cos \theta_{0}}} \mathbf{I}_{1}^{g} \\
\mathbf{I}_{1}^{v} & =e^{\frac{-2 \sigma h_{v}}{\cos \theta_{0}}} \int_{0}^{h_{v}} e^{\frac{2 \sigma z^{\prime}}{\cos \theta_{0}}} \mathbf{T}_{v} d z^{\prime} \\
\mathbf{I}_{1}^{g} & =\int_{0}^{h_{v}} \delta\left(z^{\prime}\right) e^{\frac{2 \sigma z^{\prime}}{\cos \theta_{0}}} \mathbf{T}_{g} d z^{\prime}=\mathbf{T}_{g} .
\end{aligned}
$$

The polarimetric interferometric matrix $\boldsymbol{\Omega}_{12}$ is modeled according to

$$
\begin{aligned}
\mathbf{\Omega}_{12} & =e^{j \phi_{2}} \mathbf{I}_{2}^{v}+e^{j \phi_{1}} e^{\frac{-2 \sigma h_{v}}{\cos \theta_{0}}} \mathbf{I}_{2}^{g} \\
\mathbf{I}_{2}^{v} & =e^{\frac{-2 \sigma h_{v}}{\cos \theta_{0}}} \int_{0}^{h_{v}} e^{j \kappa_{z} z^{\prime}} e^{\frac{2 \sigma z^{\prime}}{\cos \theta_{0}}} \mathbf{T}_{v} d z^{\prime} \\
\mathbf{I}_{2}^{g} & =\mathbf{T}_{g} .
\end{aligned}
$$

The exploration of the vertical dimension of the scatterer under study is performed through the complex interferometric correlation coefficient. In case of PolInSAR data, it is also possible to determine the dependency of this coefficient with polarimetry

$$
\rho\left(\mathbf{w}_{1}, \mathbf{w}_{2}\right)=\frac{\mathbf{w}_{1}^{H} \boldsymbol{\Omega}_{12} \mathbf{w}_{2}}{\sqrt{\mathbf{w}_{1}^{H} \mathbf{T}_{11} \mathbf{w}_{1} \cdot \mathbf{w}_{2}^{H} \mathbf{T}_{22} \mathbf{w}_{2}}} .
$$

where the unitary vectors $\mathbf{w}_{1}$ and $\mathbf{w}_{2}$, represent generalized scattering mechanisms. In case of the RVoG model, (11) reduces to

$$
\rho(\mathbf{w})=\frac{\mathbf{w}^{H} \boldsymbol{\Omega}_{12} \mathbf{w}}{\mathbf{w}^{H} \mathbf{T}_{11} \mathbf{w}},
$$

as equal scattering mechanisms are assumed. In [1], it was observed that the linear behavior of $\rho(\mathbf{w})$ with respect to $\mathbf{w}$ may be employed to retrieve the different parameters that characterize a forest, under the assumption of the RVoG coherent scattering model. From this study, one may see that there is not a single scattering mechanism where the volume or the ground scattering contributions are canceled, that is, it is not possible to create an interferogram which phase depends only on the ground topography. In general, the phase of any interferogram presents a vegetation bias, that as indicated in (12), may be modulated through the polarimetric scattering mechanism, that depends basically on the SAR system parameters and on the forest morphology. Even at low frequencies, such as P-band, data are affected by the vegetation bias, despite the penetration properties of microwaves at this frequency.

\section{TOPOGRAPHY ESTIMATION}

As it is evident from (8), the polarimetric interferometric covariance matrix results from the combination of the ground and the volume scattering contributions. Hence, $\boldsymbol{\Omega}_{12}$ may be written as indicated in (13) where one may observe that all the matrix entries present a dependency on the ground and the volume scattering contributions. Nevertheless, if one considers the elements elements $\boldsymbol{\Omega}_{12}(1,2)$ and $\boldsymbol{\Omega}_{12}(2,1)$, it may be seen that the phase of these elements depend only on the ground scattering contribution due to the full azimuthal symmetry of the volume scattering contribution. These phases present the same interferometric contribution from the location of phase center associated to the ground scattering center, that is, $e^{j \phi_{1}}$. Nevertheless, the polarimetric contribution through the term $t_{12}$, see (3), present opposite signs. Consequently, if one consider the product of both off-diagonal terms, it may be writhen as follows

$$
\boldsymbol{\Omega}_{12}(1,2) \boldsymbol{\Omega}_{12}(2,1)=e^{j 2 \phi_{1}} e^{-2 \frac{2 \sigma h_{v}}{\cos \phi_{0}}} m_{g}^{2}\left|t_{12}\right|^{2}
$$

where it can be observed that [3]

$$
\phi_{1}=\frac{1}{2} \arg \left\{\boldsymbol{\Omega}_{12}(1,2) \boldsymbol{\Omega}_{12}(2,1)\right\} .
$$




$$
\boldsymbol{\Omega}_{12}=e^{j \phi_{1}} e^{-\frac{2 \sigma h_{v}}{\cos \left(\theta_{0}\right)}}\left[\begin{array}{ccc}
C_{v} m_{v} e^{j\left(\phi_{2}-\phi_{1}\right)}+m_{g} & m_{g} t_{12} & 0 \\
m_{g} t_{12}^{*} & C_{v} \eta m_{v} e^{j\left(\phi_{2}-\phi_{1}\right)}+m_{g} t_{22} & 0 \\
0 & 0 & C_{v} \eta m_{v} e^{j\left(\phi_{2}-\phi_{1}\right)}+m_{g} t_{33}
\end{array}\right]
$$

Consequently, the previous two expressions make possible to have access to the undelying ground phase, associated to the ground topography, without the effect of the volume bias. As it is evident, (15) codes topography information in the range $[-\pi / 2, \pi / 2)$, so it introduces an additional wrapping in the topographic phase.

The additional wrapping may be easily solved if one considers the polarimetric matrix $\mathbf{T}_{11}$ In one considers the off-diagonal elements $\mathbf{T}_{11}(1,2)$ and $\mathbf{T}_{11}(2,1)$ it is possible to observe that these terms do not present an interferometric phase, whereas the polarimetric contribution, in terms of phase, is the same as in the case of the terms $\Omega_{12}(1,2)$ and $\boldsymbol{\Omega}_{12}(2,1)$. Consequently, considering the combination of the off-diagonal elements of the matrix $\Omega_{12}$ together with the off-diagonal elements of $\mathbf{T}_{11}$, the addition phase wrapping is eliminated by

$$
\boldsymbol{\Omega}_{12}(1,2) \mathbf{T}_{11}(2,1)=e^{j \phi_{1}} e^{-2 \frac{2 \sigma h_{v}}{\cos \phi_{0}}} m_{g}^{2}\left|t_{12}\right|^{2}
$$

where it can be observed that [3]

$$
\phi_{1}=\arg \left\{\boldsymbol{\Omega}_{12}(1,2) \mathbf{T}_{12}(2,1)\right\} .
$$

Eq. (18) provides the topographic phase in the original phase range $[-\pi, \pi)$.

Both expressions, (15) and (18), are able to provide a closed analytical expression, under the assumption of the RVoG scattering model, for the unambiguous retrieval of the underlying ground topographic phase in case of forested areas, without the necessity to perform a least squares line fit [1].

\section{RESULTS}

Eqs. (15) and (18) are equivalent with respect to the retrieval of the topographic phase. Hence, results shall be provided in the case of (18).

In order to validate (18), PolInSAR data has been simulated according the the RVoG coherent scattering model. In this case, the forest parameters are fixed to: $h_{v}=15$ $\mathrm{m}, \eta=0.25$ and $\sigma=0.3 \mathrm{~dB} / \mathrm{m}$. The ground scattering contribution is simulated according to the X-Bragg scattering model considering a flat, rough, loamy terrain with 2.2 water content. Finally, a nominal ground-to-volume ratio $m_{g} / m_{v}=-5 \mathrm{~dB}$ has been imposed. The different simulated data sets present a variation of the topographic phase $\phi_{1}\left(\phi_{2}=\phi_{1}\right.$ has been assumed $)$ with the following values $\phi_{1} \in\{-3 \pi / 4,-\pi / 2,-\pi / 4,0 \pi / 4, \pi / 2,3 \pi / 4\}$ rad in order to simulate different topographic heights. Fig. 2 details the histograms of the retrieved topographic phases considering

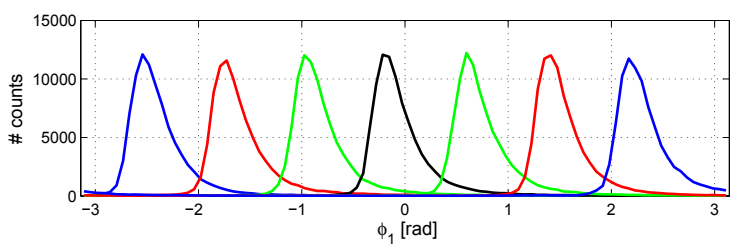

(a) Estimated $\phi_{1}$ histograms

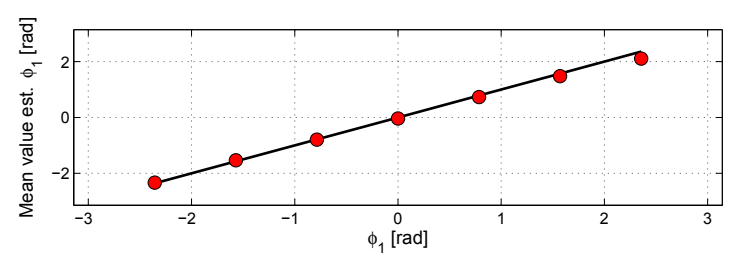

(b) Estimated $\phi_{1}$ mean value

Fig. 2: Estimated $\phi_{1}$ phase with simulated PolInSAR data.

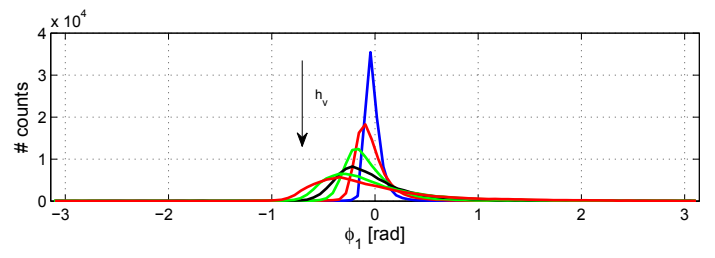

(a) Estimated $\phi_{1}$ histograms

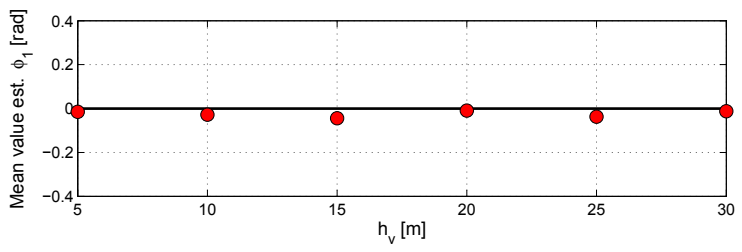

(b) Estimated $\phi_{1}$ mean value

Fig. 3: Estimated $\phi_{1}$ phase with simulated PolInSAR data.

(18), together with the corresponding mean values against the simulated topographic phase values. As one may observe, the proposed expression is able to retrieve the correct topographic information, without the bias due to the volume contribution. The performance of the previous expression to retrieve the topographic information remains contant in all the phase range and no wrapping problems are observed as the topographic phase may be retrieved in the range $[-\pi, \pi)$.

In a second set of simulations, the topographic phase is constant with a value of $\phi_{1}=0 \mathrm{rad}$, whereas the forest height varies in the range $h_{v}=\{5,10,15,20,25,30\} \mathrm{m}$. Fig. 3 details the retrieved histograms together with the corresponding mean value of the retrieved topographic phase. Again, 


$$
\mathbf{T}_{11}=e^{-\frac{2 \sigma h_{v}}{\cos \theta_{0}}}\left[\begin{array}{ccc}
m_{v} \frac{\cos \theta_{0}}{2 \sigma}\left(e^{\frac{2 \sigma h_{v}}{\cos \theta_{0}}}-1\right)+m_{g} & m_{g} t_{12} & 0 \\
m_{g} t_{12}^{*} & m_{v} \eta \frac{\cos \theta_{0}}{2 \sigma}\left(e^{\frac{2 \sigma h_{v}}{\cos \theta_{0}}}-1\right)+m_{g} t_{22} & 0 \\
0 & 0 & m_{v} \eta \frac{\cos \theta_{0}}{2 \sigma}\left(e^{\frac{2 \sigma h_{v}}{\cos \theta_{0}}}-1\right)+m_{g} t_{33}
\end{array}\right]
$$

topography is correctly estimated. Nevertheless, despite the volume contribution does not introduce a phase bias, it introduces a decorrelation factor that induces a degradation of the retrieved topographic phase. This effect may be observed in (18).

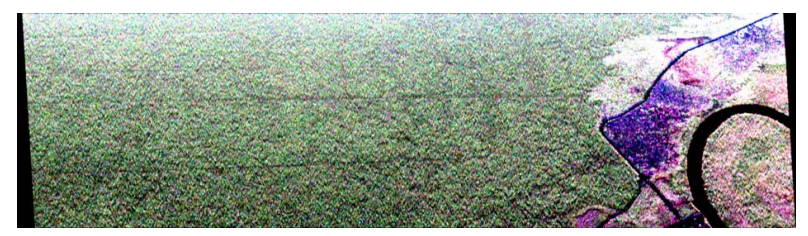

Fig. 4: Pauli RGB decomposition of the Master data set $(R=$ $\left.\left|S_{h h}-S_{v v}\right|, G=\sqrt{2}\left|S_{h v}\right|, B=\left|S_{h h}+S_{v v}\right|\right)$

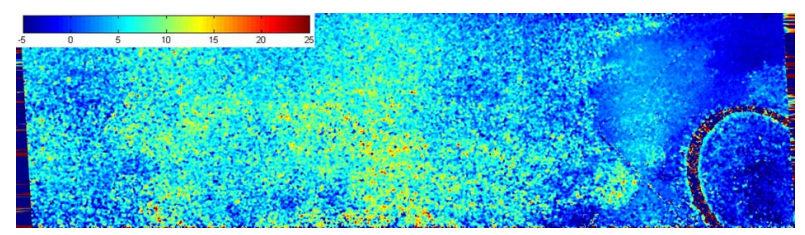

(a) Estimated underneath topography in meters

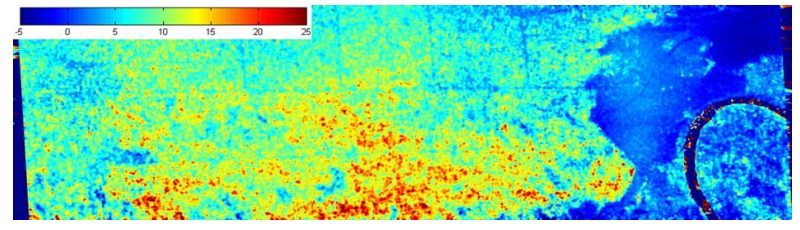

(b) Height corresponding to $S_{h v, 1} S_{h v, 2}^{*}$ in meters

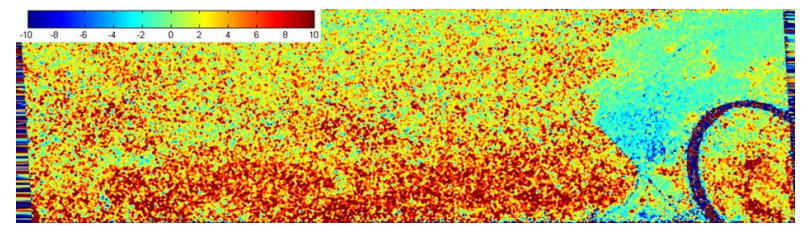

(c) Height difference in meters

Fig. 5: Indrex-II P-band data set with $15 \mathrm{~m}$ interferometric baseline.

Additionally, an evaluation of (18) to retrieve the underlying ground topography based on experimental PolInSAR has been considered. These data correspond to the second Indonesian Airborne Radar Experiment (INDREX-II), that was conducted in 2004 on the Kalimantan island of Indonesia. A P-band PolInSAR data set, with an interferometric baseline of $15 \mathrm{~m}$, has been considered where the Pauli RGB decom- position is presented in Fig. 4. As one may observe, most of the data set corresponds to tropical forest, whereas on the right-hand side an sparsely vegetated area and a river may be observed. Fig. 5 presents the estimated underneath topographic height, the height corresponding to the interferogram $S_{h v, 1} S_{h v, 2}^{*}$ and the corresponding phase difference. Since the phase center associated to $S_{h v, 1} S_{h v, 2}^{*}$ may be assumed to be the highest or close to the highest one, the height difference presented in Fig. 5 is consequent with this argument. Additionally, one may compare the retrieved topography on the sparsely vegetated area (right-hand side) against the topography obtained in the forested one. As it may observed, the height variation in the transition between both areas is more diffuse in the case of the retrieved underneath topographic height, confirming that topography is correctly retrieved. Additionally, the height difference for the sparsely forested area obtained from the difference of the retrieved topographic height $\phi_{1}$ and the height corresponding to the interferogram $S_{h v, 1} S_{h v, 2}^{*}$ is close to zero, whereas this difference presents an approximate mean value of $7 \mathrm{~m}$ in the case of the forested area.

\section{CONCLUSIONS}

As demonstrated, the analysis of the RVoG coherent scattering model has made possible to derive two analytical expressions, based on PolInSAR data, that allow a direct and unambiguous estimation of the underlying ground topography without the bias induced by the vegetation cover in case of forested areas. Results based on both, simulated as well as experimental PolInSAR data confirm the validity of these expressions.

\section{REFERENCES}

[1] S. Cloude and K. Papathanassiou, "Three-stage inversion process for polarimetric SAR interferometry," Radar, Sonar and Navigation, IEE Proceedings -, vol. 150, no. 3, pp. 125-134, 2003.

[2] S. R. Cloude and K. P. Papathanassiou, "Polarimetric SAR interferometry," Geoscience and Remote Sensing, IEEE Transactions on, vol. 36, no. 5, pp. 1551-1565, Sep. 1998.

[3] C. López-Martínez and K. P. Papathanassiou, "Procedimiento para la estimación de la topografía de la superfície de la tierra en áreas con cobertura vegetal," Spanish Patent Request P201 000 793, June 16, 2010. 